\title{
Malignant change in a benign pulmonary hamartoma
}

\section{ANTONIO BASILE, ALFREDO GREGORIS, BENIAMINO ANTOCI, MASSIMO ROMANELLI}

\author{
From the Divisione di Medicina Interna, Ospedale Generale, San Vito al Tagliamento-Pordenone, and the \\ Istituto di Anatomia Patologica, Ospedale Regionale, Udine, Italy
}

ABSTRACT Sarcomatous transformation in a longstanding, clinically silent hamartoma is reported. Malignant change occurred shortly after resection, suggesting that surgical manipulation may have played a part.

Hamartomas are benign tumours of the lung, consisting of connective tissue with cleft like spaces lined by epithelial cells. ${ }^{2}$ In most instances the connective tissue component is predominantly cartilagenous; but myxoid, leiomyomatous, lipomatous, or mixed variants also occur. Malignant change is extraordinarily rare, and the authenticity of many published cases is doubtful. ${ }^{3}$

We report a patient with a hamartoma of the right lung that remained clinically silent and radiologically unchanged for 37 years. Its resection coincided with an explosive sarcomatous evolution.

\section{Case report}

In October 1986 a 62 year old woman was admitted with chest pain and non-productive cough. In 1949 she had been investigated for hemoptysis, and a chest radiograph taken at the time had shown a partially calcified round mass the size of an orange in the right lower lobe. A diagnosis of calcified hydatid cyst had been made, and no medical or surgical treatment was carried out. She had had an extrauterine pregnancy in 1954 and a hysterectomy for fibroids in 1972, and had had asthma and bronchitis for several years.

Clinically, there was dullness and reduced ventilation at the right base; electrocardiography and routine laboratory tests showed no abnormality. At thoracocentesis a small quantity of viscous fluid containing red cells and mesothelial cells was obtained. Chest radiography and computed tomography showed a moderate effusion on the right side, with a large calcified mass with the same characteristics as the lesion seen on the chest radiograph in 1949.

At thoracotomy in December 1986 the right lower lobe was found to be almost totally replaced by a hard mass, and the surrounding parenchyma was compressed to $1-3 \mathrm{~cm}$ thickness. There were fibrous adhesions to the diaphragm but no signs of tumour extension outside the lung. The lower lobe was resected.

Gross examination of the specimen disclosed a lobular,

Address for reprint requests: $\operatorname{Dr}$ A Basile, Divisione Medica, Ospedale Civile, 33078 San Vito al Tagliamento, Italy.

Accepted 14 December 1988 well circumscribed, and partially cystic mass $12 \mathrm{~cm}$ in $\vec{x}$ diameter with calcified walls. Histologically, there was a $f$ mixture of loose fibromyxoid, connective, and adipose tissue; $\dot{\omega}$ foci of cartilage with areas of bony metaplasia; lymphaticin vessels; and occasional duct like structures with an epithelial ${ }_{N}^{\omega}$ lining (fig 1). Although the cartilage sometimes appeared too be immature, there was no evidence of malignant change.

Postoperatively the patient complained of increasing $\frac{-}{3}$ weakness, but nothing untoward was found on clinical or radiological examination. At the end of February 1987 she $\bar{\supset}$ was readmitted with increasing weakness, tachycardia, and dyspnoea. Radiologically, there was an opacity in the lower $\varrho_{\infty}$ right thorax, extending to the hilum and superior medias- $\bullet$ tinum; computed tomography showed that the opacity was solid, and was causing a leftward shift of the trachea ando heart. The patient's cardiocirculatory function rapidly deteriorated and she died on 13 March 1987.

At necropsy a fleshy, reddish white tumour occupied the

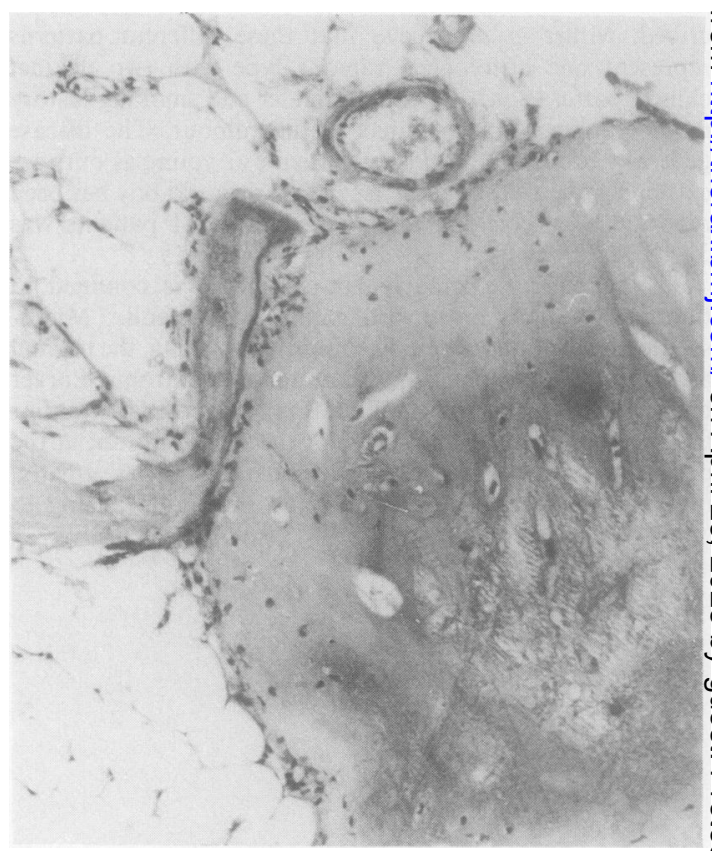

Fig 1 Part of the tumour removed at thoracotomy. This field consists of mature cartilage and adipose tissue. (Haematoxylin and eosin.) 


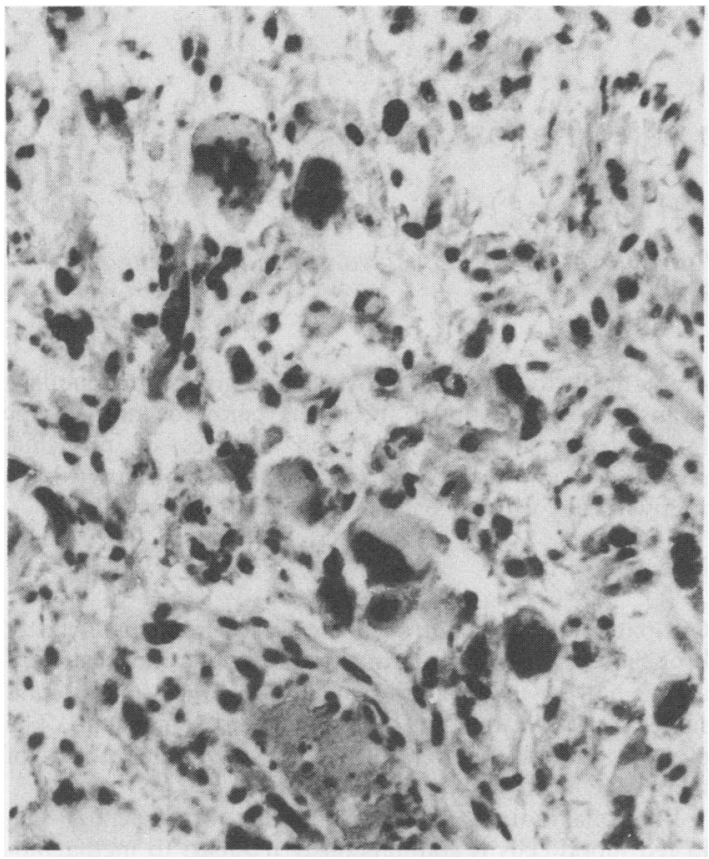

Fig 2 Pleomorphic round, spindle, and giant cells in the necropsy specimen. (Haematoxylin and eosin.)

lower right thoracic cavity, and extended into the middle and upper lobe at the hilum. The right pleura, mediastinum, anterior thoracic wall, and sternum were infiltrated, and tumour had spread along the diaphragm to the thoracotomy scar. A large thrombus was found in the right atrium and old emboli in branches of the pulmonary artery.

Histologically, the tumour consisted of spindle cells and medium sized or large round cells, arranged in poorly oriented bundles. Many cells were multinucleated and bizarre (fig 2). The immunoperoxidase technique produced patchy staining for vimentin; but cytokeratin, myoglobin, carcinoembryonic antigen, epithelial membrane antigen, $\alpha_{1}$ chymotrypsin, lysozyme, human chorionic gonadotrophin, factor VIII, and S-100 protein were not present. The appearances were those of a primary pleomorphic sarcoma of either cartilagenous or connective tissue origin.

\section{Discussion}

Most hamartomas are less than $4 \mathrm{~cm}$ across, but some may reach $20 \mathrm{~cm}$ in diameter; calcification or ossification may be present. Histologically, there is a cartilagenous and an epithelial component in apposition to adipose tissue, and myxoid fibrous tissues. ${ }^{2}$ Although the cartilage may appear immature or proliferating, these tumours are almost invariably benign, and easily distinguished from pulmonary blastoma and carcinosarcoma.

The question of malignant degeneration in pulmonary hamartomas remains controversial, and some pathologists would deny that it occurs. Hayward and Carabasi ${ }^{3}$ reported a hamartoma with adenocarcinomatous change in a woman of 58 , and reviewed 12 previously published cases. Nine were unacceptable because of incomplete documentation or incorrect diagnosis; only three were thought to be possible examples of hamartomas with carcinomatous or sarcomatous transformation, but their exact nature was doubtful.

The present case supports the idea that hamartomas are mesenchymal neoplasms capable of malignant change. In this instance it seems likely that the malignancy originated in areas of neoplastic tissue that were not evident at operation. Malignant degeneration followed almost immediately after resection. This may, of course, have occurred in the absence of surgical intervention; but the long period of clinical and radiological inactivity, the initially benign appearance of the tumour, and its subsequent aggressive behaviour less than three months later all suggest a causal relation.

A rather similar case was reported in 1955 by Kuyer. ${ }^{4} \mathrm{~A}$ benign neoplasm, consistent in appearance with a hamartoma, was excised from an 18 year old boy who subsequently developed a metastasising sarcoma. The period of observation, however, was short, and clinical and pathological details were scanty. ${ }^{3}$ The basis of malignant change in such cases remains obscure, but stimulation by surgical trauma or disruption of immunological tolerance may be important.

\section{References}

1 Carter D, Eggleston J. Atlas of tumor pathology. Fascicle 17. Washington DC: Armed Forces Institute of Pathology, 1980:213-50.

2 Anonymous. Case records of the Massachusetts General Hospital (case 45-1986). N Engl J Med 1986;315:1277-85.

3 Hayward RH, Carabasi RJ. Malignant hamartoma of the lung: fact or fiction? J Thorac Cardiovasc Surg 1967;53:457-66.

4 Kuyer PJ. En geval van maligne degenererend long hamartoon. Ned Tijdschr Geneeskd 1955;99(part 1): 1884-6. 